\title{
Transistor por Efeito de Campo e Fotocondutor de Poli(0-Metoxianilina).
}

\author{
Roberto K. Onmori, Luiz Henrique C. Mattoso e Roberto M. Faria
}

Resumo: Esse artigo apresenta resultados sobre fabricação e caracterização de um transistor por efeito de campo (FET) tendo a poli(o-metoxianilina) - POMA - como material ativo. Uma adaptação de processos de microeletrônica tradicional foi feita para a confecção desse dispositivo. O FET desenvolvido apresentou modulação por voltagem de porta $\mathrm{V}_{\mathrm{g}}$, mas operou mesmo em $\mathrm{V}_{\mathrm{g}}=0$. A corrente de dreno $\mathrm{I}_{\mathrm{D}}$ aumentou significativamente sob iluminação na região da radiação visível e infravermelho próximo, mostrando o caráter fotocondutor da POMA dopada e, portanto, do dispositivo. Um modelo teórico baseado nos mecanismos de condução eletrônica da POMA, e de efeitos de interface metal-polímero foi elaborado, ajustando-se muito bem aos resultados experimentais.

Palavras-chave: Dispositivos eletrônicos, transistor por efeito de campo, fotocondutividade, polimeros condutores, aplicações.

\section{Introdução}

Os materiais poliméricos estão entre os que apresentam maiores resistividades elétricas, sendo os que apresentam propriedades térmicas e mecânicas mais versáteis. Há cerca de vinte anos, entretanto, o conceito de que esses materiais são exclusivamente isolantes elétricos foi mudado com a descoberta dos Polímeros Condutores ${ }^{[1]}$. Esses polímeros condutores são na verdade materiais isolantes, mas sob dopagem química atingem condutividades comparáveis às dos metais podendo ter sua condutividade alterada em até 15 ordens de grandeza. Passam assim de excelentes isolantes a ótimos condutores elétricos, com propriedade adicional de reverter essa condutividade adquirida desde que o material seja desdopado. Portanto, em princípio, pode-se contro- lar o valor de sua condutividade dentro de uma faixa que cobre valores entre $10^{-11} \mathrm{~S} / \mathrm{cm}$ a $10^{5} \mathrm{~S} / \mathrm{cm}^{[2,3]}$. Cada um dos polímeros condutores hoje conhecidos varre toda a faixa semicondutora, região na qual se desenvolveu a tecnologia da moderna microeletrônica. Portanto, hoje se iniciam inúmeros estudos de aplicações desses materiais enquanto semicondutores ativos, procurando levá-los ao nível de importância ocupado pelos inorgânicos Silício e Arseneto de Gálio. O primeiro polímero condutor foi o poliacetileno. Este foi sintetizado pela primeira vez em 1955 tendo como produto um pó escuro insolúvel, porém ainda não condutor. Cerca de vinte anos mais tarde no Instituto de Tecnologia de Tókio ele foi obtido acidentelmente em forma de uma película elástica brilhante ${ }^{[2,4]}$. Em $1977^{[1,2]}$, na Universidade da Pensilvânia, estudos sobre dopagem química e propriedades elétricas com

Roberto K. Onmori - Laboratório de Microeletrônica, Escola Politécnica da USP, Av. prof. Luciano Gualberto, trav. $3 n^{\circ} 158$, Cidade Universitária, CEP: 05508900, São Paulo, SP; Luiz Henrique C. Mattoso - Centro Nacional de Pesquisa e Desenvolvimento de Instrumentação Agropecuária, CNPDIA/EMBRAPA, C.P. 741, São Carlos, CEP: 13560-970, SP; Roberto M. Faria - Instituto de Física de São Carlos/USP, C.P. 369, São Carlos, CEP: 13560-970, SP. 
essas películas resultaram por descobrir a propriedade da condutividade elétrica do poliacetileno. Outros polímeros ${ }^{[2,4]}$ apresentaram a mesma propriedade, tendo eles em comum a estrutura conjugada da cadeia molecular, isto é, alternância entre ligações químicas duplas e simples ao longo da cadeia. Entre eles se destacam: o polipirrol, o politiofeno, o poli(parafenilineo), etc.

Logo após a descoberta desses "plásticos eletrônicos" deu-se início a busca de aplicações na área de microeletrônica procurando-se fabricar diodos, transistores e outros dispositivos optoeletrônicos e fotônicos. Entretanto essas aplicações têm esbarrado na alta instabilidade que as propriedades elétricas desses plásticos apresentam, quando em contato com $\mathrm{o}$ ar, e na dificuldade de controlar adequadamente os valores requeridos de condutividade. Mesmo assim vários protótipos de dispositivos eletrônicos foram feitos e publicados na literatura especializada ${ }^{[5-16]}$, com curvas características de corrente elétrica $I_{D} \mathrm{em}$ função da tensão de dreno $V_{D}$ semelhantes às dos dispositivos inorgânicos comerciais ${ }^{[6,7]}$. Outras aplicações, também oriundas da variação reversível da condutividade adquirida, já têm sido amplamente divulgadas, destacando-se as aplicações em baterias $^{[2]}$, em recobrimento antiestático ${ }^{[17]}$, em proteção contra corrosão ${ }^{[18]}$, em transdutores ${ }^{[19]}$, em biosensores $^{[20]}$ e em sensores de gases ${ }^{[21]}$.

Em meados da década de 80 um novo polímero condutor se destaca, a Polianilina ${ }^{[22-24]}$. Sua estrutura, diferentemente dos demais, não era conjugada, e seu mecanismo de dopagem não se dá pelo processo de oxi-redução, mas por protonação da cadeia polimérica. Suas vantagens sobre os demais plásticos eletrônicos advêm da facilidade de síntese e processamento de filmes e, principalmente, da sua melhor estabilidade quando em presença do ar, tanto no estado não-dopado quanto no dopado. Logo a polianilina (PANI) e seus derivados se candidatam como materiais potencias a inúmeras aplicações, dentre elas as na área de dispositivos eletrônicos.

O presente trabalho, desenvolvido nos laboratórios do Grupo de Polímeros do Instituto de Física de São Carlos e no Laboratório de Microeletrônica da Escola Politécnica $^{[25]}$, ambos da Universidade de São Paulo, aplicou um dos derivados da PANI, a poli(ometoxianilina) (POMA), na fabricação de um transistor por efeito de campo, semelhante a um tradicional. Nesse trabalho, apresentamos toda a adaptação da tecnologia usual de microeletrônica usada na fabrica- ção de dispositivos à base de silício para o transistor polimérico, e os resultados da caracterização do dispositivo e sua modelação teórica. Esse trabalho tem o mérito de apresentar pela primeira vez um dispositivo por efeito de campo utilizando a POMA. Além disso, observou-se para este dispositivo a propriedade adicional de alterar sensivelmente o sinal característico de corrente de dreno por iluminação, mostrando um forte caráter fotocondutor. Até o momento, a PANI tem sido pouco aplicada enquanto material ativo em microeletrônica. Recentemente ${ }^{[12]}$ foi feito um dispositivo retificador à base de polianilina, numa estrutura Alumínio/PANI/ITO (vidro recoberto com óxido de Índio e Estanho), sem qualquer estudo de modulação por campo, ou de fotocondução.

\section{Parte Experimental}

\section{O Processo de Fabricação do Transistor}

O transistor de POMA foi construído sobre uma lâmina de silício monocristalino, orientação $<100>$, fortemente dopado (tipo p) com resistividade em torno de $1 \Omega$.cm e com uma das faces polida. A lâmina de silício serviu, além de suporte mecânico para o dispositivo, também como eletrodo por onde se aplica a tensão de porta $\mathrm{V}_{\mathrm{G}}$. Foi feita uma adaptação de processos de microeletrônica tradicional para a confecção do dispositivo, uma vez que determinadas etapas de preparação de substratos e de fotogravação eram agressivas ao material polimérico, e que o processo de dopagem química da POMA atacava a estrutura do fotoresiste. A fabricação do dispositivo pode ser dividida em diferentes etapas, descritas a seguir:

Etapa 1: - Limpeza da lâmina - Nessa etapa foram removidos os diversos tipos de impurezas e compostos orgânicos adsorvidos na superfície, assim como eventuais óxidos formados na lâmina que surgem durante o tempo de armazenamento. A limpeza foi feita com $\mathrm{H}_{2} \mathrm{SO}_{4} / \mathrm{H}_{2} \mathrm{O}_{2}$ (3:1); $\mathrm{H}_{2}$ Odeionizada $/ \mathrm{H}_{2} \mathrm{O}_{2}$ / $\mathrm{NH}_{4} \mathrm{OH}(5: 1: 1)$ e finalmente com $\mathrm{HF} / \mathrm{H}_{2} \mathrm{O}(1: 50)$.

Etapa 2: - Formação da camada de óxido - A lâmina foi colocada em forno de oxidação térmica, de modo que a superfície do silício fique em contato com um fluxo de oxigênio à temperatura de $1000^{\circ} \mathrm{C}$. $\mathrm{O}$ oxigênio reage quimicamente com o silício formando o óxido de silício - $\mathrm{SiO}_{2}$. A espessura do óxido tem aproximadamente $40 \mathrm{~nm}$. O óxido crescido 
serve como isolante elétrico entre o silício e o polímero que será posteriormente depositado sobre o óxido, e deve ser de boa qualidade para suportar altos campos elétricos provenientes da tensão $\mathrm{V}_{\mathrm{G}}$.

Etapa 3: - Deposição dos eletrodos - Essa etapa consiste na fabricação dos eletrodos de dreno e fonte, onde é aplicada a diferença de potencial $V_{D}$ e medida a corrente característica $\mathrm{I}_{\mathrm{D}}$, que atravessa o polímero que será depositado. Para esse processo usamos a técnica de fotolitografia denominada Liftoff. Esse processo envolve o desenho dos contatos elétricos e a transferência de sua imagem para a lâmina de silício. A geometria dos contatos elétricos (Figura 1) foi desenhada no programa denominado de AutoCad, versão 10, do equipamento Pattern Generator - IMAGE 100. A distância entre os eletrodos varia de 10,20 e $30 \mu \mathrm{m}$ e o comprimento do canal é de $2 \mathrm{~cm}$. Essa geometria é ideal para as medidas elétricas, onde a pequena distância entre eletrodos e o grande comprimento do canal favorecem o ganho da corrente elétrica. Para transferir a imagem no silício, uma camada de material fotosensível (fotoresiste AZ 1350 J Develop) foi depositado. O Pattern Generator, através de seu sistema óptico, transfere o desenho sensibilizando o fotoresiste. Após a revelação, com o produto químico AZ 350 da Develop, o fotoresiste sensibilizado foi removido, ficando essas regiões expostas. Uma camada de ouro de espessura $20 \mathrm{~nm}$ foi evaporada sobre toda a superfície da lâmina de silício oxidada. O fotoresiste ao ser removido carrega consigo todo ouro depositado sobre a sua superfície deixando somente o ouro depositado diretamente no silício oxidado.

Etapa 4: - Deposição do polímero - A POMA na forma de base de esmeraldina, sintetizada quimicamente conforme descrito na literatura ${ }^{[22,25]}$, foi dissolvida em clorofórmio na proporção de $1 \mathrm{~g}$ para cada $100 \mathrm{ml}$ de solvente. A técnica usada para a deposição e obtenção de um filme fino de POMA foi o spin coating. Nesse processo a solução de POMA em clorofórmio é gotejada sobre a superfície do substrato até que o lí-

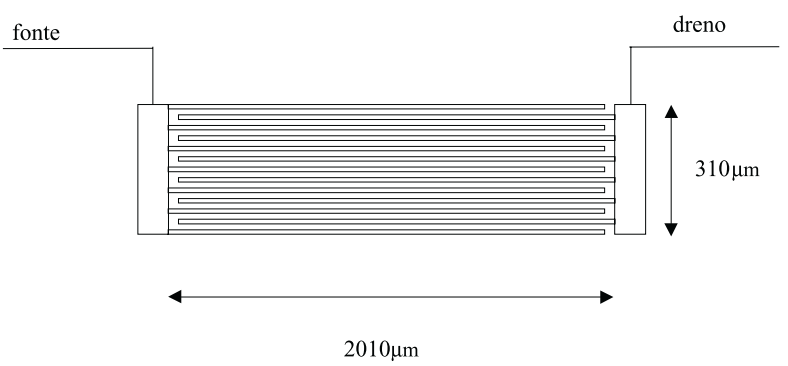

Figura 1 - Esquema dos contatos elétricos utilizados como dreno e fonte.

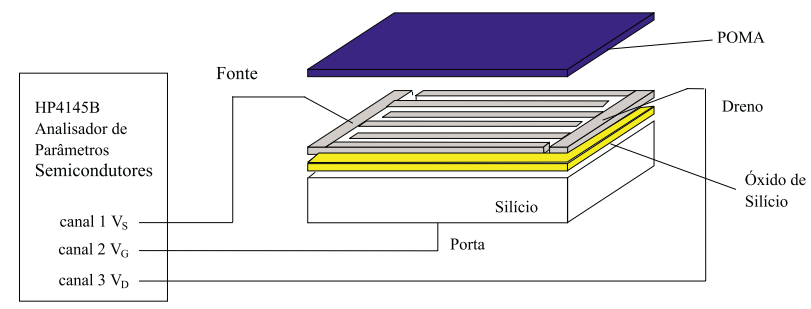

Figura 2 - Esquema da montagem do transistor.

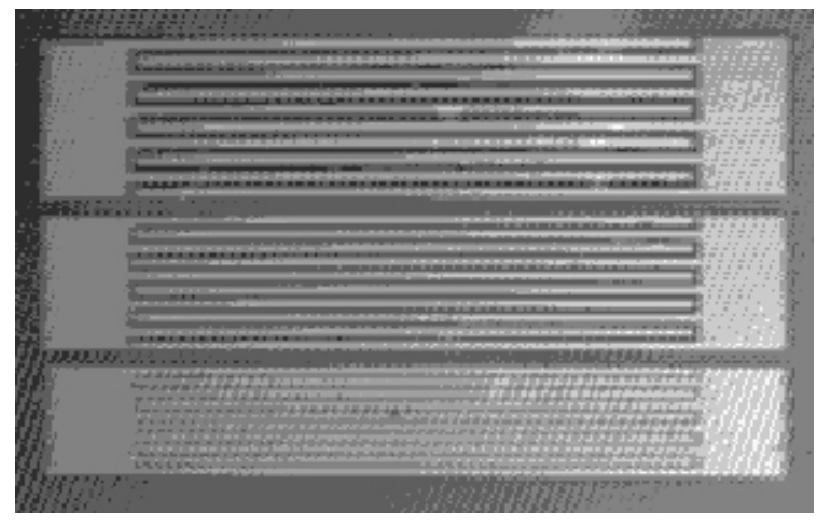

Figura 3 - Foto do transistor a base de POMA

quido depositado molhe homogeneamente a superfície de óxido de silício. Em seguida a lâmina é colocada em rotação a $1000 \mathrm{rpm}$ por 30 segundos. O clorofórmio (solvente) evapora rapidamente assim obtém-se uma fina camada de POMA, em torno de 20 nm. A figura 2 mostra o esquema da montagem do transistor.

Etapa 5: - Dopagem da POMA - A POMA tal como depositada sobre a lâmina do dispositivo é um material isolante. Para dopá-la, e assim obter uma condutividade típica de um semicondutor, se imerge todo o dispositivo numa solução de ácido clorídrico 1,0 molar por 10 segundos. Logo em seguida, com um jato de nitrogênio, retira-se o excesso de ácido ao mesmo tempo em que se seca o filme de POMA. Obtemos, finalmente o transistor à base de POMA cuja foto é mostrada na figura 3 .

\section{Caracterização do dispositivo}

A caracterização elétrica desse dispositivo foi feita utilizando-se o equipamento "analisador de parâmetros semicondutores - HP 4145B" e uma estação de trabalho Went Worth com micromanipuladores para posicionar corretamente as pontas de prova. A figura 2 ilustra também o esquema de ligações para a obtenção da curva corrente $\mathrm{I}_{\mathrm{D}} v s$ tensão $\mathrm{V}_{\mathrm{D}}$ do transistor. A existência do efeito fotocondutor foi investigada 
através de medidas de corrente $v s$ tensão no dispositivo irradiado sob diferentes intensidades de iluminação.

\section{Resultados e Discussão}

A caracterização elétrica do dispositivo de POMA para diferentes tensões de porta é ilustrado na figura 4. Os resultados experimentais mostram muito bem o comportamento clássico dos dispositivos por efeito de campo, iniciando em corrente zero para $\mathrm{V}_{\mathrm{D}}=0$, aumentando com a tensão, e atingindo um patamar de corrente para tensões mais elevadas. Uma diferença básica é percebida nos valores iniciais de corrente. Nas curvas obtidas com os dispositivos de POMA uma retificação aparente é registrada, provavelmente devido ao efeito de uma região de depleção (carga espacial) que surge na interface polímero-metal gerando assim uma barreira tipo Schottky. A figura 5 ilustra medidas para $\mathrm{V}_{\mathrm{G}}=0 \mathrm{sob}$ diferentes intensidades de irradiação luminosa na faixa do visível e IV próximo. A radiação luminosa interage com a estrutura do material, provocando excitações eletrônicas nas faixas onde existem absorções ópticas no material. Essas absorções promovem os elétrons (portadores de carga) dos níveis mais localizados para os mais energéticos onde os portadores são mais deslocalizados, e portanto possuem maior mobilidade.

\section{Modelo teórico}

O princípio de operação de dispositivos FET tradicionais (JFET e MESFET) se baseia na evolução de uma camada de inversão - região de depleção formada na interface entre o eletrodo de porta e o semicondutor dopado que é o material ativo do dispositivo. O mecanismo de condução, traduzido pela

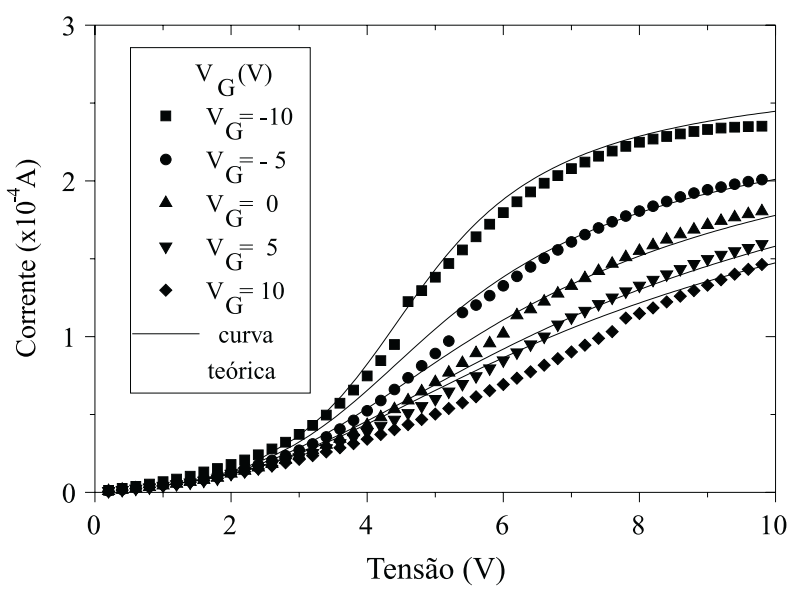

Figura 4. Curva corrente vs. tensão, experimental e teórica, para o transistor fabricado a base de POMA.

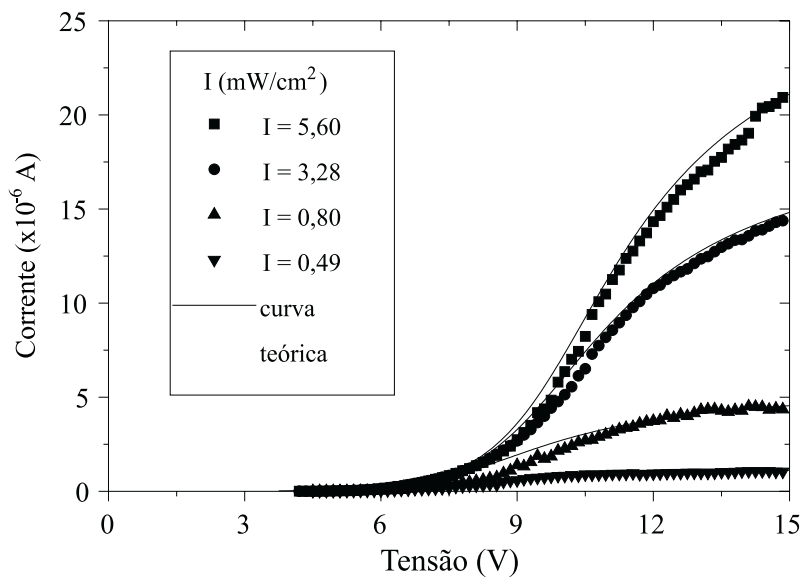

Figura 5. Curva corrente $v s$. tensão, experimental e teórica, do transistor a base de POMA, $\mathrm{V}_{\mathrm{G}}=0$, sob várias intensidades de iluminação.

curva característica $\mathrm{I}_{\mathrm{D}} v s . \mathrm{V}_{\mathrm{D}}$, é devido a portadores majoritários e sua dinâmica é controlada pela espessura dessa camada de inversão, que tem a função de estrangular o canal de condução dos portadores majoritários. Para um valor de voltagem de porta $\mathrm{V}_{\mathrm{G}}$ fixo, a corrente $I_{D}$ atinge a saturação $I_{D s}$ a partir de um valor $\mathrm{V}_{\mathrm{Ds}}$, que é denominado voltagem de saturação. Para $\mathrm{V}_{\mathrm{G}}=0, \mathrm{I}_{\mathrm{Ds}}$ tem seu valor máximo, $\mathrm{I}_{\mathrm{p}}$, e é denominada de corrente de pinch off. Esse fenômeno acontece para um determinado valor de tensão junto à interface semicondutor-eletrodo de dreno designado por $\mathrm{V}_{\mathrm{p}}$, e nessa voltagem o estrangulamento do canal junto ao eletrodo de dreno é total ${ }^{[26]}$.

No dispositivo polimérico apresentado acima, os fenômenos físicos responsáveis pelas curvas características não são os mesmos do citado acima. No caso da poli(o-metoxianilina) dopada com $\mathrm{HCl}$ os portadores são os buracos ${ }^{[27]}$, e a condução se faz por todo o volume do material não sendo necessários o conceito de canal de condução e de camada de inversão. Nesse caso, supondo que os contatos sejam ôhmicos, ou pelo menos que não sejam bloqueantes, uma vez aplicada a tensão $\mathrm{V}_{\mathrm{D}}$ no dispositivo, surgirá como resposta a corrente $I_{D}$. Para explicar as curvas ilustradas na Figura 4, para diferentes tensões $\mathrm{V}_{\mathrm{G}}$, apresentamos um modelo detalhado a seguir, fazendo de início duas considerações, uma sobre a mobilidade dos portadores e outra sobre sua velocidade de arraste: $i$ ) a mobilidade varia segundo o eixo $y$ definido ao longo de uma perpendicular ao eletrodo de porta, sendo a mobilidade maior junto à interface polímero-óxido, e menor na superfície que fica exposta ao ambiente; $i$ ) a velocidade varia com o campo, seja pelo fato de que para baixos campos, sendo o material altamente desordenado, os portadores encontram muitos obstáculos à sua migração devido à sua baixa energia cinética, seja pelo efeito de retrodifusão 
térmica, que é um efeito de corrente de difusão estimulada termicamente de sentido oposto à corrente de arraste $^{[28]}$. Uma aproximação que faremos diz respeito à uniformidade do campo elétrico ao longo do eixo $x$ (direção definida pela linha que liga os eletrodos de fonte e de dreno). Isto significa que a concentração de portadores não varia ao longo de $x$. A voltagem de porta quando negativa aumenta os valores de $\mathrm{I}_{\mathrm{D}}$ e quando positiva age no sentido inverso, como visto na figura 4 . Dentro do modelo, $\mathrm{V}_{\mathrm{G}}<0$ atrairia os portadores para junto da interface polímero-óxido, onde a mobilidade, segundo nossas considerações é maior; e para $\mathrm{V}_{\mathrm{G}}>0$, os portadores são então arrastados em direção à superfície do filme polimérico onde a mobilidade é menor.

Em geral os filmes poliméricos são mais desordenados em suas superfícies livres onde também adsorve impurezas com certa facilidade. Já a superfície que fica em contato com um substrato tende a ser mais ordenada e protegida de contaminações. Portanto, é razoável a suposição de um "gradiente" de mobilidade ao longo de $y$.

\section{Cálculo}

A figura 6 ilustra um esquema do dispositivo polimérico e os eixos $x, y$ e $z$ são definidos segundo as direções dadas pela distância entre os eletrodos fonte $\mathrm{e}$ dreno, pela normal ao plano do dispositivo e pelo comprimento dos eletrodos. A corrente elétrica que atravessa o polímero $\mathrm{I}_{\mathrm{D}}$ - ao longo do eixo $x$ - em resposta à $\mathrm{V}_{\mathrm{D}}$ pode ser calculada a partir da equação diferencial:

$$
\mathrm{dI}=\mathrm{JdA}
$$

onde $\mathrm{J}=\rho \mathrm{v}$ é a densidade de corrente e $\mathrm{dA}=\mathrm{Zdy}$ um diferencial de área no interior do material polimérico no plano $y z$, sendo $Z$ a espessura do dispositivo. $\rho$ é a densidade de portadores do material e $\checkmark$ a velocidade de arraste desses portadores.

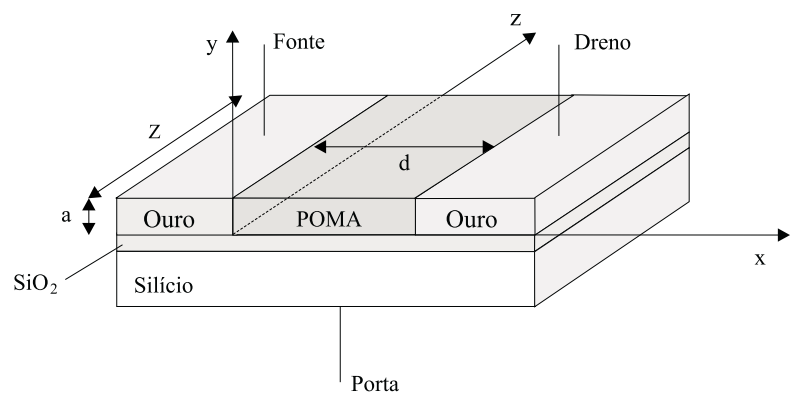

Figura 6. Estrutura do Transistor e suas dimensões para modelamento.
A consideração $i$ ) feita acima sobre a velocidade de arraste $v$ pode ser expressa matematicamente por:

$$
\mathrm{v}=\frac{\mu \mathrm{E}}{1+\frac{\mu \mathrm{E}}{\mathrm{v}_{\mathrm{s}}}}
$$

onde $E=\mathrm{V}_{\mathrm{D}} / L$ ( $L$ é a distância entre os eletrodos dreno e fonte), $\mu$ é a mobilidade e $v_{s}$ a velocidade de saturação dos portadores.

Para efeito de cálculo podemos expressar a variação da mobilidade dos portadores ao longo do eixo $y$, conforme a consideração $i$ ), segundo a relação linear:

$$
\mu=\mu_{0}\left(1-\frac{\mathrm{k}}{\mathrm{a}} \mathrm{y}\right)=\mu_{0}(1-\mathrm{by})
$$

$\operatorname{com} 0 \leq \mathrm{k} \leq 1$ e $\mathrm{b}=\frac{\mathrm{k}}{\mathrm{a}}$.

Sendo os buracos os portadores móveis, a tensão $\mathrm{V}_{\mathrm{G}}$ quando positiva os expulsa em direção à superfície da mostra, e quando negativa os atrai concentrando-os junto a interface polímero-óxido. Também para simplificar os cálculos, podemos expressar a densidade de portadores numa relação linear $\operatorname{com} y$, como segue:

$$
\rho=\chi_{0} y+\rho_{1}
$$

Ambos, $\chi_{0}$ e $\rho_{1}$ são constantes e $\chi_{0}$ depende de $V_{G}$.

Juntando-se as Eqs. 1 a 4, podemos escrever então a seguinte equação de corrente para o dispositivo:

$$
\mathrm{dI}=\left(\chi_{0} \mathrm{y}+\rho_{1}\right) \frac{(1-\text { by }) \mu_{0} \mathrm{E}}{1+\frac{(1-\mathrm{by}) \mu_{0} \mathrm{E}}{\mathrm{v}_{\mathrm{s}}}} \mathrm{Zdy}
$$

ou ainda,

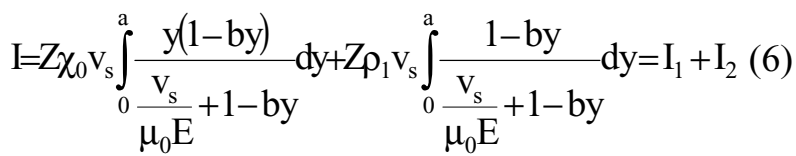

As soluções das integrais $\mathrm{I}_{1}$ e $\mathrm{I}_{2}{ }^{[25]}$ levam aos respectivos resultados:

$$
I_{1}=a \frac{\mu_{0} E v_{s}}{v_{s}+\mu_{0} E} \quad \text { e } \quad I_{2}=\left(\frac{a^{3}}{2}+\frac{k^{3}}{3}\right)\left(\frac{1}{\left(\frac{v_{s}}{\mu_{0} E}+1\right)}\right)
$$


e a corrente do transistor será, portanto, dada por:

$$
I_{\text {tr }}=Z a\left[a\left(\frac{1}{2}+\frac{k}{3}\right) \chi_{0}+\rho_{1}\right]\left(\frac{\mu_{0} E}{1+\frac{\mu_{0} E}{v_{s}}}\right)
$$

A expressão acima não ajusta, entretanto, as curvas experimentais na região de $V_{D}$ baixos. Isto se deve provavelmente ao fato de termos desprezado os efeitos de interface colocando o campo uniforme ao longo de $x$. Para corrigir, ao menos parcialmente essa falha, adicionamos ao modelo um diodo em série com $o$ transistor, e a tensão $V_{D}$ fica sendo a soma da tensão do transistor $\mathrm{V}_{\text {tr }}$ mais a do diodo $\mathrm{V}_{\mathrm{d}}$. A corrente é a mesma pois a associação é em série. Como na interface metal-polímero ocorre o efeito de junção Schottky, a corrente do diodo pode ser descrita pela corrente Schottky:

$$
I_{d}=I_{o}\left[\exp \left(\frac{q V_{d}}{K T}\right)-1\right]
$$

A partir da consideração de que a corrente que flui pelo diodo deverá ser a mesma daquela que flui pelo transistor temos:

$I_{d}=I_{t r} \Rightarrow I_{0}\left[\exp \left(\frac{q V_{d}}{K T}\right)-1\right]=Z a\left[a\left(\frac{1}{2}+\frac{k}{3}\right) \chi_{0}+\rho_{1}\right]\left(\frac{\mu_{0} V_{\text {tr }}}{d+\frac{\mu_{0} V_{t r}}{V_{s}}}\right)(10)$

Aplicando-se o logarítimo nas equações, e isolando o termo $\mathrm{V}_{\mathrm{d}}$, tem-se:

$$
V_{d}=\frac{K T}{q} \ln \left\{\frac{Z a}{I_{o}}\left[a\left(\frac{1}{2}+\frac{k}{3}\right) \chi_{0}+\rho_{1}\right]\left(\frac{\mu_{0} V_{t r}}{d+\frac{\mu_{0} V_{t r}}{v_{s}}}\right)\right\}
$$

Substituindo a equação (11) na equação (10), obtemos a equação geral da corrente $\mathrm{I}_{\mathrm{tr}}$ em função de $\mathrm{V}_{\text {tr }}$, que é a característica $\mathrm{I}_{\mathrm{D}} v s \mathrm{~V}_{\mathrm{D}}$, lembrando-se que uma pequena parte da tensão $V_{D}$ fica aplicada nas interfaces:

$$
I_{\text {tr }}=I_{0}\left[\exp \left(\ln \left\{\frac{Z a}{I_{o}}\left[a\left(\frac{1}{2}+\frac{k}{3}\right) \chi_{0}+\rho_{1}\right]\left(\frac{\mu_{0} V_{\text {tr }}}{d+\frac{\mu_{0} V_{\text {tr }}}{v_{\mathrm{s}}}}\right)\right\}+1\right)-1\right]
$$

Tabela 1. Parâmetros de ajuste das curvas de corrente versus tensão do transistor.

\begin{tabular}{ccccc}
\hline$(\mathrm{V})$ & $\chi_{0}\left(\mathrm{C} / \mathrm{cm}^{2}\right)$ & $\rho_{1}\left(\mathrm{C} / \mathrm{cm}^{3}\right)$ & $\mu_{0}\left(10^{-5} \mathrm{~cm}^{2} / \mathrm{V} . \mathrm{s}\right)$ & $\nu_{\mathrm{s}}\left(10^{-2} \mathrm{~cm} / \mathrm{s}\right)$ \\
\hline $\mathrm{V}_{\mathrm{G}}=-10$ & 417 & 0,4 & 8,5 & 3 \\
$\mathrm{~V}_{\mathrm{G}}=-5$ & 259 & 0,26 & 5,5 & 4,3 \\
$\mathrm{~V}_{\mathrm{G}}=0$ & 210 & 0,26 & 3,5 & 5,9 \\
$\mathrm{~V}_{\mathrm{G}}=5$ & 199 & 0,21 & 2,5 & 6 \\
$\mathrm{~V}_{\mathrm{G}}=10$ & 192 & 0,2 & 2,4 & 5,9 \\
\hline
\end{tabular}

Tabela 2. Parâmetros de ajuste para o transistor sob iluminação

\begin{tabular}{ccc}
\hline$\left(\mathrm{mW} / \mathrm{cm}^{2}\right)$ & $\chi_{0}\left(\mathrm{C} / \mathrm{cm}^{2}\right)$ & $\rho_{1}\left(10^{-3} \mathrm{C} / \mathrm{cm}^{3}\right)$ \\
\hline $\mathrm{I}=5,60$ & 15 & 16 \\
$\mathrm{I}=3,28$ & 12 & 11 \\
$\mathrm{I}=0,80$ & 3,1 & 3,2 \\
$\mathrm{I}=0,49$ & 0,72 & 0,75 \\
$\mathrm{I}=0,40$ & 0,29 & 0,32 \\
\hline
\end{tabular}

As figuras 4 e 5 também apresentam ajustes da expressão (12) sobre os resultados experimentais. Como pode-se observar, para baixas tensões aparece um efeito tipo retificador na curva IxV. Esse efeito é típico de queda de voltagem na junção, e para resolvêlo supusemos uma equação Schottky (Equação 9). Considerando-se a complexidade dos mecanismos de migração de portadores no interior do polímero, essa equação é uma boa aproximação, embora estudos ainda possam ser feitos para se aperfeiçoar este modelo teórico. Os parâmetros de ajuste são $\chi_{0}, \rho_{1}, \mu_{\mathrm{o}}$ e $v_{\mathrm{s}}$, tem seus valores mostrados na Tabela 1 , para $\mathrm{I}_{\mathrm{o}}=7,5$ $10^{-7}$ (A) e $\mathrm{k}=0,1$ para o transistor. No caso do transistor sob iluminação (Tabela 2, com $\mathrm{I}_{\mathrm{o}}=2,110^{-10}$ (A) e $\mathrm{k}=0,1)$ os valores de $\mu_{\mathrm{o}}=910^{-5} \mathrm{~cm}^{2} / \mathrm{V}$.s e de $\mathrm{n}_{\mathrm{s}}$ $=810^{-2} \mathrm{~cm} / \mathrm{s}$ permanecem constantes. $\mathrm{Na}$ avaliação de dispositivos eletrônicos, esses valores são considerados bons e o valor da mobilidade dos portadores está em concordância com os dados $\left(\mu_{0} \cong 10^{-4} \mathrm{~cm}^{2} /\right.$ v.s) obtidos na literatura ${ }^{[27]}$.

\section{Conclusão}

A contribuição mais importante desse trabalho foi mostrar que a poli(o-metoxianilina), adequadamente dopada, é um material ativo para a fabricação de um transistor fotocondutor modulado por campo. Uma das etapas mais árduas, e que foi vencida, foi evitar as interferências dos processamentos litográficos e de dopagem do polímero, uma vez que o tratamento 
físico-químico de um era nocivo ao outro. No entanto, as etapas de fabricação do dispositivo ainda podem ser melhoradas, e sua proteção por encapsulamento deverá aumentar seu tempo de vida útil. Pelo valor relativamente baixo da mobilidade dos portadores nesse polímero, esse dispositivo não é considerado rápido e, portanto, não poderá vir a ser aplicado em dispositivos chaveadores, ou amplificadores, na área de comunicações ou em sistemas de microondas. Este, entretanto, pode compor aplicações na área de sensores, principalmente de gases, e de fotodispositivos. A busca de sensores de gases eficientes e de baixo custo é relativamente recente e está ligada diretamente com pesquisas em Ciência Ambiental. Também na medicina dispositivos orgânicos poderão desempenhar um papel importante, mas hoje esses polímeros encontram sucesso enquanto dispositivos tradicionais como diodos e dispositivos eletroluminescentes.

\section{Agradecimentos}

Os autores agradecem à FAPESP e ao CNPq pelo apoio financeiro.

\section{Referências Bibliográficas}

1. Chiang, C.K.; Fincher, C.R.; Park, Y.W.; Heeger, A.J.; Shirakawa, H.; Louis, E.J.; Grau, S.C.; Macdiarmid, A.G.; Phys. Rev. Lett. 39,1098 (1977).

2. Kaner, R.B.; Macdiarmid, A.G.; Scient. Am. 60, Feb. (1988).

3 Adams, P.N.; Laughlin, P.J.; Monkman, A.P.; Solid State Comm. 91, 875 (1994).

4. Mattoso, L.H.C. "Plásticos que conduzem eletricidade: ficção ou realidade?" matéria publicada na revista Polímeros: Ciência e Tecnologia, no 3 ano VI, jul/set, p. 6 (1996).

5. Stubb, H.; Punkka, E.; Paloheimo, J. Materials Science and Engineering, 10(3) (1993).

6. Paloheimo, J.; Punkka, E.; Stubb, H.; Kuivalainen, P. In: lower-Dimensional systems adn Molecular Electronics, editado por Metzger, R.M. et at. Plenum Press, New York, p. 635 (1991).
7. Paloheimo, J.; Kuivalainen, P.; Stubb, H.; Vuorimaa, E.; Yli-Lahti, P. Appl. Phys. Lett. 56 (12), 1157 (1990).

8. Dodabalapur, A.; Katz, H.E.; Torsi, L.; Haddon, R.C. Science, 269, 1560 (1995).

9. Hwang, S.W.; Yu, Y.S.; Ha, W.I.; Kim, T.G.; Han, C.K.; Park, J.H.; Kim, M.S.; Kim, E.K.; Min, S.K. Appl. Phys. Lett. 69 (13), 1924 (1996).

10. Burroughes, J.H.; Jones, C.A.; Friend, R.H. Nature, 335, 137 (1988).

11. Willander, M.; Assadi, A.; Svensson, C. Synth. Met., 55-57, 4099 (1993).

12. Chen, S-A.; Fang, Y. Synth. Met. 60, 215 (1993).

13. Salih., A.J.; Haynes, D.M.; Hepburn, A.R. Synth. Met. 71 (1-3), 2257, 1995.

14. Takashima, W.; Kaneko, M.; Dkaneto, K.; MacDiarmid, A.G. Synth. Met. 71 (1-3), 22645 (1995).

15. MacDiarmid, A.G. Proceed. International Conference on Science and Technology of Synthetic Metals, Utah, Estados Unidos, Julho (1996) p.7.

16. Mello, J.C.; Tessler, N.; Pichler, K., Friend, R.H. Proceed. International Conference on Science and Technology of Synthetic Metals, Utah, Estados Unidos, Julho (1996) p.406.

17. Angelopoulos, M.; Patel, N.; Gelorme, J.; Shaw, J.M. Proceedings of the International Conference on Science and Technology of Synthetic Metals Seoul-Coréia, Jun., (1994) p.451.

18. Wessling, B.; Adv. Mat. 6, 222 (1994).

19. Kaneto, K.; Kaneko, M.; Min, Y.; Macdiarmid, A.G.; Synth. Met. 71, 2211 (1995).

20. Deshpande, M.V.; Malnerkar, D.P.; Prog. Polym. Sci. 18, 623 (1993).

21. Braker, P.S.; Monkman, A.P.; Petty, M.C. Proceed. International Conference on Science and Technology of Synthetic Metals, Utah, Estados Unidos, Julho (1996) p.409.

22. Mattoso, L.H.C. \& MacDiarmid, A.G. Oxidation States of Polyanilines, In: J.C. Salamone, The 
Polymeric Materials Encyclopedia, CRC Press Inc., v.7, p.5505 (1996).

23. Mattoso, L.H.C. Polianilinas: síntese, estrutura e propriedades. Química Nova, 19(4), 1-12 (1996).

24. Huang, W.S., Humphrey, B.D. \& MacDiarmid, A.G. J. Chem. Soc. Faraday Trans. I. 82, 23852400 (1986).

25. Onmori, K.O. "Fabricação, caracterização e modelamento de um dispositivo de efeito de campo à base de poli(o-metoxianilina) " tese de doutoramento, Escola Politécnica-USP, São Paulo, (1997).

26. Sze, S.M.; Physics of Semiconductor Devices, Cap. VI, 2ª Ed., John Wiley \& Sons (1981).

27. Megulhão, S.; Faria, R.M.; Leal Ferreira, G. F. \& Sworakowski J. Chem. Phys. Lett. (no prelo).

28. Badareu E. \&Popescu, I. Gaz Ionisés, Cap. I, Editions Dunod (1965). 